\title{
Modelagem para previsão/estimação: uma aplicação Neuro-Fuzzy
}

\author{
Luiz Carlos Benini, José Gilberto Spasiani Rinaldi \\ Universidade Estadual Paulista - Departamento de Estatística \\ 19060-900, Campus Presidente, SP \\ E-mail: benini@fct.unesp.br, gilberto@ fct.unesp.br
}

\begin{abstract}
Resumo: Neste trabalho utiliza-se de um sistema de inferência fuzzy que incorpora o aprendizado por meio de um conjunto de dados onde as variáveis de entrada estão relacionadas com suas respectivas variáveis de saídas. Takagi e Sugeno foram pioneiros em propor um modelo de sistema de inferência fuzzy, onde são utilizadas entradas e regras fuzzy mas com a saída do sistema sendo conjuntos não-fuzzy. Este modelo é uma excelente ferramenta para modelagem de problemas complexos e não lineares, quando combinado com a estrutura da rede de Sistema de Inferência Fuzzy Neuro Adaptativa (ANFIS). Sistemas de inferência fuzzy são utilizados com base em um conjunto regras, do tipo "se...então", que representam as entradas e saídas do modelo, associando a capacidade de aprendizado das redes neurais artificiais com o poder de interpretação lingüístico dos sistemas de inferência fuzzy, as quais são capazes de tratar conhecimentos incompletos, incertos ou mesmo conflitantes. É apresentado uma aplicação onde os dados estimados pelo modelo se mostraram correlacionados, indicando boa exatidão para o modelo neuro-fuzzy obtido, onde pode ser verificada a capacidade e a eficiência dos sistemas de inferência fuzzy.
\end{abstract}

\section{Introdução}

As fases envolvidas com as variáveis que quantificam um processo de análise de um conjunto de dados são cercadas de imprecisões e incertezas inerentes aos próprios aspectos físicos e estruturais dos dados. Os sistemas de inferência fuzzy são usados nestes casos, pois os mesmos fazem o mapeamento de processos com entradas advindas de um conjunto de dados resultantes de observações, ou experimentações, em saídas precisas $y=f(x)$, sendo $x$ a variável de entrada, possuindo diferentes graus de imprecisão e incerteza, $y$ a saída do sistema de inferência fuzzy e $f$ uma representação quantitativa do mapeamento.

A teoria dos conjuntos fuzzy, introduzida em [4], surgiu como um meio de representação e manipulação de dados imprecisos, e pelo fato de os conjuntos clássicos apresentarem limitações para lidar com problemas onde transições de uma classe para outra acontecem de forma lenta e gradual.

Zadeh [5] generalizou a função característica de maneira que esta pudesse assumir um número infinito de valores no intervalo $[0,1]$ sugerindo que alguns elementos são mais membros de um conjunto do que outros. Neste caso o grau de pertinência pode assumir qualquer valor no intervalo [0,1]. A função que define o grau de pertinência de um determinado elemento em um conjunto fuzzy, levando em consideração o seu universo de discurso, é definida como função de pertinência.

Neste trabalho utiliza-se de um sistema de inferência fuzzy que incorpora o aprendizado por meio de um conjunto de dados onde as variáveis de entrada estão relacionadas com suas respectivas variáveis de saídas. Takagi e Sugeno [3] estão entre os pesquisadores pioneiros a proporem estruturas de sistemas de inferência fuzzy baseadas em um conjunto de padrões de ajuste para a sua definição. Sistemas de inferência fuzzy têm sido utilizados para modelar processos complexos, não lineares e vagos, com base em um conjunto regras, do tipo "se...então", que representam as entradas e saídas do modelo, e que combinadas com métodos de redes neurais artificiais, dão origem a um novo método classificado como híbrido, que associa a capacidade de aprendizado das redes neurais artificiais com o poder de interpretação linguístico dos sistemas de inferência fuzzy. 
Dentre as abordagens que usam métodos híbridos destaca-se o sistema de inferência fuzzy neuro adaptativo (ANFIS) proposto em [2], que suporta o sistema de Takagi-Sugeno de ordem zero ou um, permitindo várias variáveis de entrada com somente uma variável saída.

\section{Material e Método}

Para a construção do modelo foram consideradas sete variáveis de entrada, sendo elas: temperatura máxima, temperatura mínima, ponto de orvalho, pressão atmosférica, umidade relativa, vento velocidade, vento direção, e uma variável de saída representando a temperatura do ar.

Para o desenvolvimento do trabalho foi utilizado um conjunto de dados, com as observações advindas de uma estação meteorológica, dividido em dois grupos selecionados aleatoriamente: dados de treinamento e dados de testes para a validação do modelo, e então construídos um sistema de inferência fuzzy, do tipo Takagi-Sugeno [3], definido por um conjunto de regras da forma: se $x$ é $A$ e $y$ é $B$ então $z=f(x, y)$, sendo $x$ e $y$ variáveis fuzzy, $A$ e $B$ conjuntos fuzzy e $f$ uma função de $x$ e $y$ que aproxima o valor de $z$.

O modelo neuro-fuzzy com base no modelo do tipo de Takagi-Sugeno de primeira ordem e no algoritmo ANFIS, considerando um sistema de inferência fuzzy de duas entradas, $x_{1}$ e $x_{2}$, uma saída, $f$, e composto por duas regras fuzzy:

$$
\begin{aligned}
& \text { Regra 1: Se } x_{1} \text { é } \mathbf{A}_{1}^{1} \text { e } x_{2} \text { é } \mathbf{A}_{1}^{2} \text {, então } f_{1}=p_{1} x_{1}+q_{1} x_{2}+r_{1} \\
& \text { Regra 2: Se } x_{1} \text { é } \mathbf{A}_{2}^{1} \text { e } x_{2} \text { é } \mathbf{A}_{2}^{2} \text {, então } f_{2}=p_{2} x_{1}+q_{2} x_{2}+r_{2} \text {. }
\end{aligned}
$$

pode ser representada como na Figura 1.

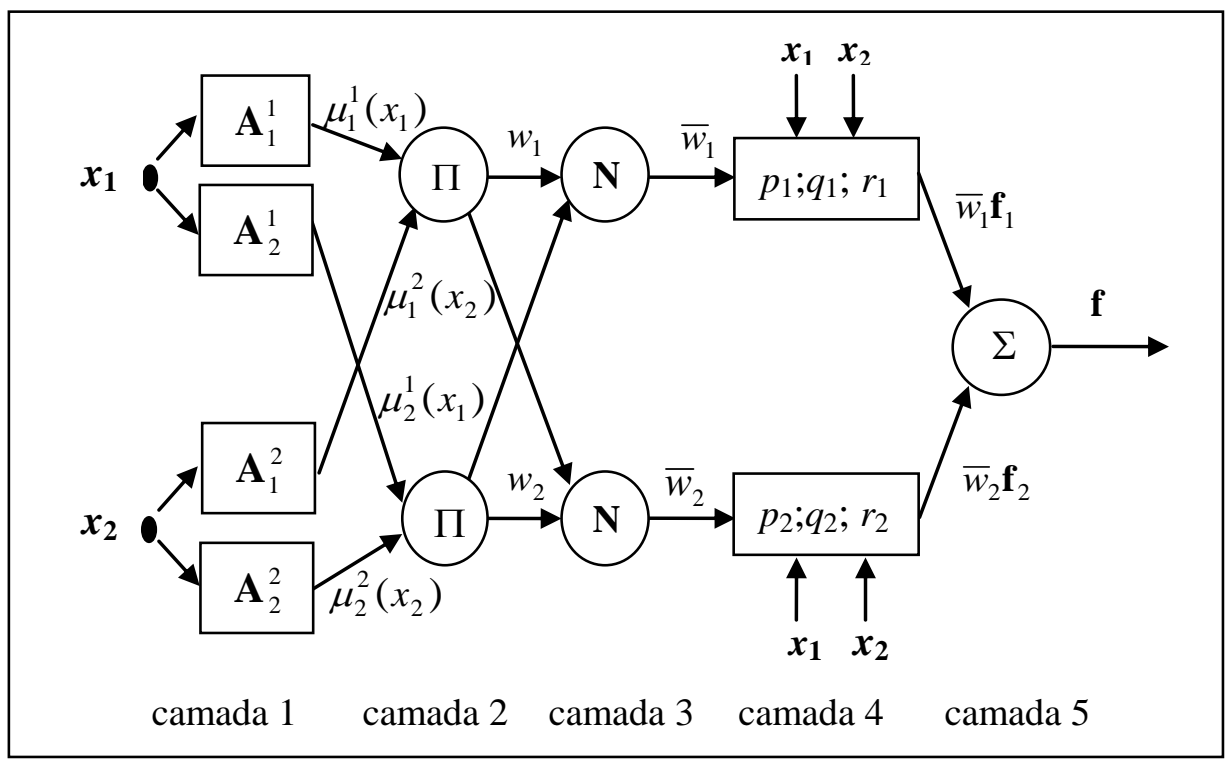

Figura 1 - Arquitetura típica de um modelo ANFIS.

$\mathrm{Na}$ estrutura do sistema de inferência fuzzy se pode, ainda, incorporar às regras um conhecimento proveniente de um especialista, dado por uma regra heurística, ao conhecimento intrínseco dos dados, obtidos a partir do processo de aprendizagem. Estas regras são ajustadas através de um processo de otimização buscando minimizar alguma medida de erro, sendo o vetor de variáveis independentes formado pelos parâmetros das funções de pertinências e os coeficientes dos consequentes das regras.

O modelo ANFIS ajusta por meio do algoritmo do gradiente descendente os parâmetros das funções de pertinências, os quais são neste trabalho do tipo gaussianas. O método dos mínimos quadrados é usado para encontrar os coeficientes da função de saída para o modelo. 
A aprendizagem da estrutura ANFIS envolve a determinação do número de funções de pertinência por variável, a obtenção de um conjunto de regras fuzzy e de um conjunto de funções lineares, por valores atribuídos a parâmetros de ajustes, denominados raios de vizinhança dos centros dos agrupamentos, por meio da técnica de agrupamento subtrativo desenvolvida em [1], particionando o espaço de entrada e saída de um conjunto de dados.

Quando não se conhece "a priori" quantos agrupamentos deve haver para um determinado conjunto de dados, o agrupamento subtrativo é um algoritmo rápido e robusto para saber este número. Esta técnica permite a localização dos centros de agrupamentos de um conjunto de dados, que é o centro da função de pertinência e, a partir destas, obter as regras fuzzy.

\section{Resultados e discussões}

A partir do conjunto dos dados e com o uso das técnicas de otimização não linear do modelo ANFIS um sistema de previsão foi implementado utilizando o conjunto de variáveis de entrada como variáveis independentes e a variável de saída a variável dependente.

A identificação do modelo escolhido foi o que apresentou o menor erro quadrático para o conjunto de treinamento como para o conjunto de teste, e se obteve um número de funções de pertinência, e em função destas, também, um número de regras que satisfizeram ao critério do erro mínimo. Foi obtido o tipo de função de pertinência na forma gaussiana e o tipo de sistema fundamentado foi o modelo fuzzy de Takagi-Sugeno de primeira ordem.

$\mathrm{Na}$ determinação do modelo foram obtidas quatro funções de pertinências para cada uma das variáveis de entrada e deste modo o modelo identificado para estimar a temperatura do ar é constituído de sete variáveis de entrada, com quatro conjuntos fuzzy associados a cada uma das variáveis de entrada, quatro regras com os antecedentes de cada uma conectados pelo operador "e" e, portanto, quatro funções lineares, cada qual, representando a parte do consequente da regra fuzzy.

\begin{tabular}{lccccccccc}
\hline Variável de Entrada & $\left(x_{i}\right)$ & $m$ & $\sigma$ & $m$ & $\sigma$ & $m$ & $\sigma$ & $m$ & $\sigma$ \\
\hline Temperatura Máxima & $\left(x_{1}\right)$ & 22.26 & 1.148 & 16 & 6.43 & 22.13 & 1.36 & 19.84 & 4.392 \\
Temperatura Mínima & $\left(x_{2}\right)$ & 21.36 & 4.168 & 20.8 & 1.301 & 16.14 & 6.383 & 20.16 & 1.409 \\
Ponto de Orvalho & $\left(x_{3}\right)$ & 15.68 & 8.531 & 12.87 & 5.128 & 3.678 & 1.822 & 19.21 & 20.28 \\
Pressão Atmosférica & $\left(x_{4}\right)$ & 956.3 & 6.77 & 959.3 & 6.102 & 954.9 & 3.377 & 956.9 & 6.9 \\
Umidade Relativa & $\left(x_{5}\right)$ & 75.94 & 18.21 & 75.77 & 14.49 & 50.04 & 12.85 & 95.18 & 10.42 \\
Vento Velocidade & $\left(x_{6}\right)$ & 2.018 & 2.648 & 4.223 & 3.651 & 1.278 & 0.3847 & 0.97 & 2.62 \\
Vento Direção & $\left(x_{7}\right)$ & 103.6 & 75.08 & 102 & 76.86 & 77.96 & 71.7 & 56.35 & 75.48 \\
\hline
\end{tabular}

Tabela 1 - Parâmetros das funções de pertinência $\left(\mu_{i}\right)$

Na Tabela 1 são apresentados os parâmetros das funções de pertinência $(\mu)$, onde o parâmetro $m$ denota o centro de um agrupamento (centro de uma função de pertinência) e $\sigma$ a variação dos dados observados com relação à sua média.

Os parâmetros dos consequentes das regras fuzzy são apresentados na Tabela 2, onde os parâmetros $c_{i}(i=1, \ldots, 8)$ são os coeficientes da função linear do consequente de cada uma das regras do modelo.

\begin{tabular}{ccccccccc}
\hline Regras & $c_{1}$ & $c_{2}$ & $c_{3}$ & $c_{4}$ & $c_{5}$ & $c_{6}$ & $c_{7}$ & $c_{8}$ \\
\hline 1 & 0.009 & 1.014 & -0.020 & -0.005 & 0.003 & -0.047 & -4.135 & 4.032 \\
2 & 0.139 & 0.881 & -0.017 & 0.003 & 0.008 & -0.046 & -0.0008 & -0.669 \\
3 & 1.570 & -0.086 & -0.502 & -0.009 & 0.155 & 0.009 & 0.0005 & -5.092 \\
4 & -0.137 & 0.516 & 0.624 & -0.004 & -0.081 & 0.007 & -0.0004 & 12.290 \\
\hline
\end{tabular}

Tabela 2. Parâmetros das funções lineares de saída dos consequente 
Com os parâmetros da função de pertinência, as regras e as funções lineares que definem as saídas para os consequentes de cada regra, são as seguintes:

Regra 1: Se $x_{1} \in[18.8,25.7], x_{2} \in[8.9,26.9], x_{3} \in[-2.2,22.7], x_{4} \in[9941,976.6]$,

$$
\begin{aligned}
& x_{5} \in[24.0,95.0], x_{6} \in[0,5.9], x_{7} \in[1,328.8], \text { então } \\
& y_{1}=0.01 x_{1}+1.01 x_{2}-0.02 x_{3}-0.01 x_{4}+0.01 x_{5}-0.05 x_{6}-4.14 x_{7}+4.03
\end{aligned}
$$

Regra2: Se $x_{1} \in[4.2,27.5], x_{2} \in[16.9,24.7], x_{3} \in[-2.5,22.7], x_{4} \in[941,969.6]$,

$$
\begin{aligned}
& x_{5} \in[32.3,95.0], x_{6} \in[0,5.9], x_{7} \in[1,332.6], \text { então } \\
& y_{2}=0.14 x_{1}+0.88 x_{2}-0.02 x_{3}+0.01 x_{4}+0.01 x_{5}-0.05 x_{6}-0.001 x_{7}-0.67
\end{aligned}
$$

Regra 3: Se $x_{1} \in[18.05,26.21], x_{2} \in[0,26.9], x_{3} \in[-1.8,9.1], x_{4} \in[944.7,965]$,

$$
\begin{aligned}
& x_{5} \in[11.5,88.6], x_{6} \in[0.1,2.4], x_{7} \in[1,293.1], \text { então } \\
& y_{3}=1.57 x_{1}-0.09 x_{2}-0.50 x_{3}-0.01 x_{4}+0.16 x_{5}+0.01 x_{6}+0.0005 x_{7}-5.09
\end{aligned}
$$

Regra 4: Se $x_{1} \in[6.6,27.5], x_{2} \in[15.9,24.4], x_{3} \in[-2.2,22.7], x_{4} \in[941.0,969.6]$,

$$
\begin{aligned}
& x_{5} \in[63.92,95.44], x_{6} \in[0.0,5.9], x_{7} \in[1,282.79], \text { então } \\
& y_{4}=-0.14 x_{1}+0.52 x_{2}+0.62 x_{3}-0.01 x_{4}-0.08 x_{5}+0.01 x_{6}-0.0004 x_{7}+12.29
\end{aligned}
$$

Na Tabela 3 são apresentados os dados observados da temperatura do ar e as estimativas obtidas pelo modelo neuro-fuzzy, juntamente com os erros relativos os quais são utilizados para medir a precisão entre estes valores.

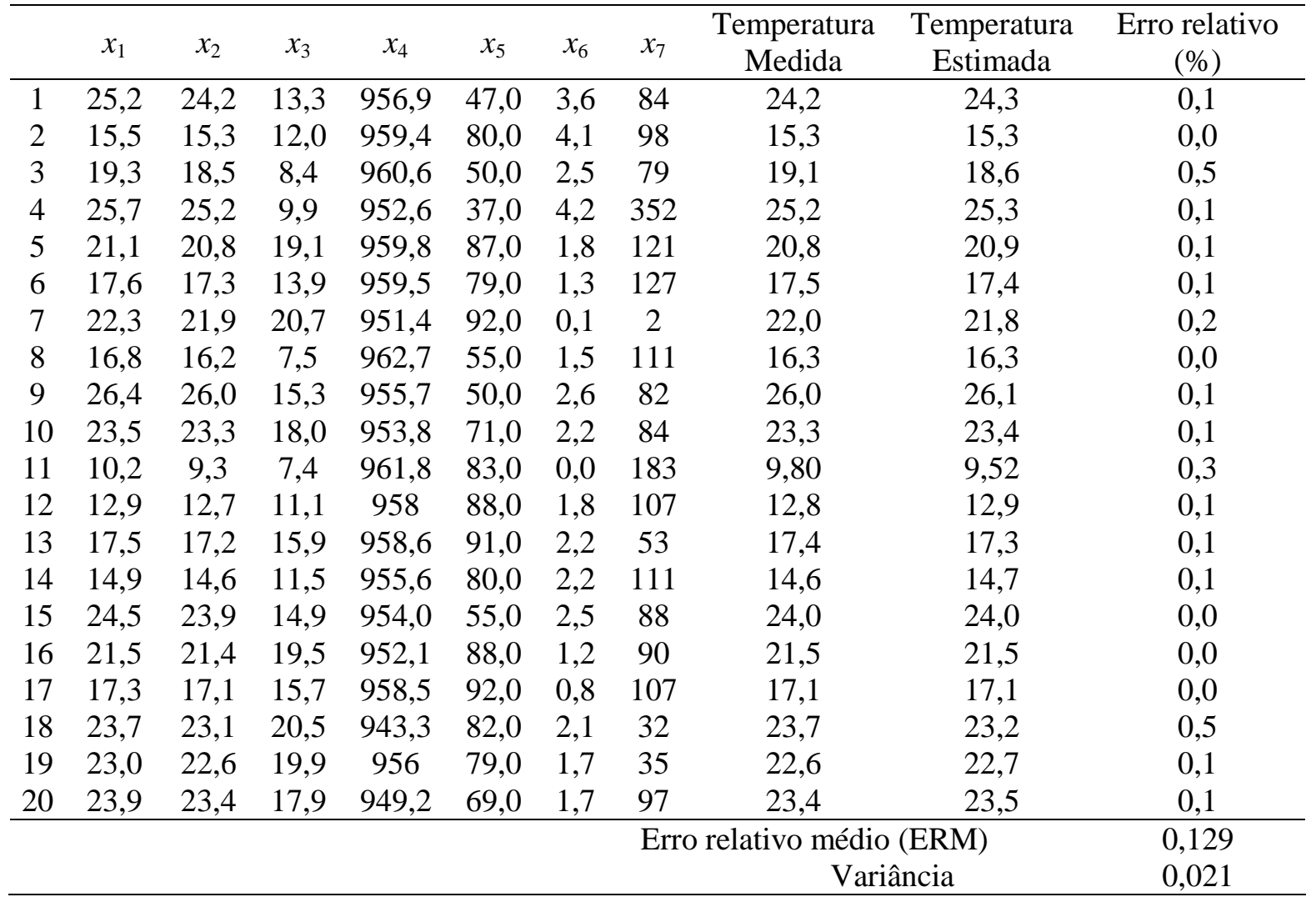

Tabela 3 - Resultados do modelo para os dados da temperatura do ar $\left({ }^{\circ} \mathrm{C}\right)$ 
Na Figura 2 são mostrados os gráficos dos dados medidos (observados) e os valores estimados pela modelagem fuzzy, onde se pode verificar o comportamento do modelo neurofuzzy para os valores dos dados estimados.

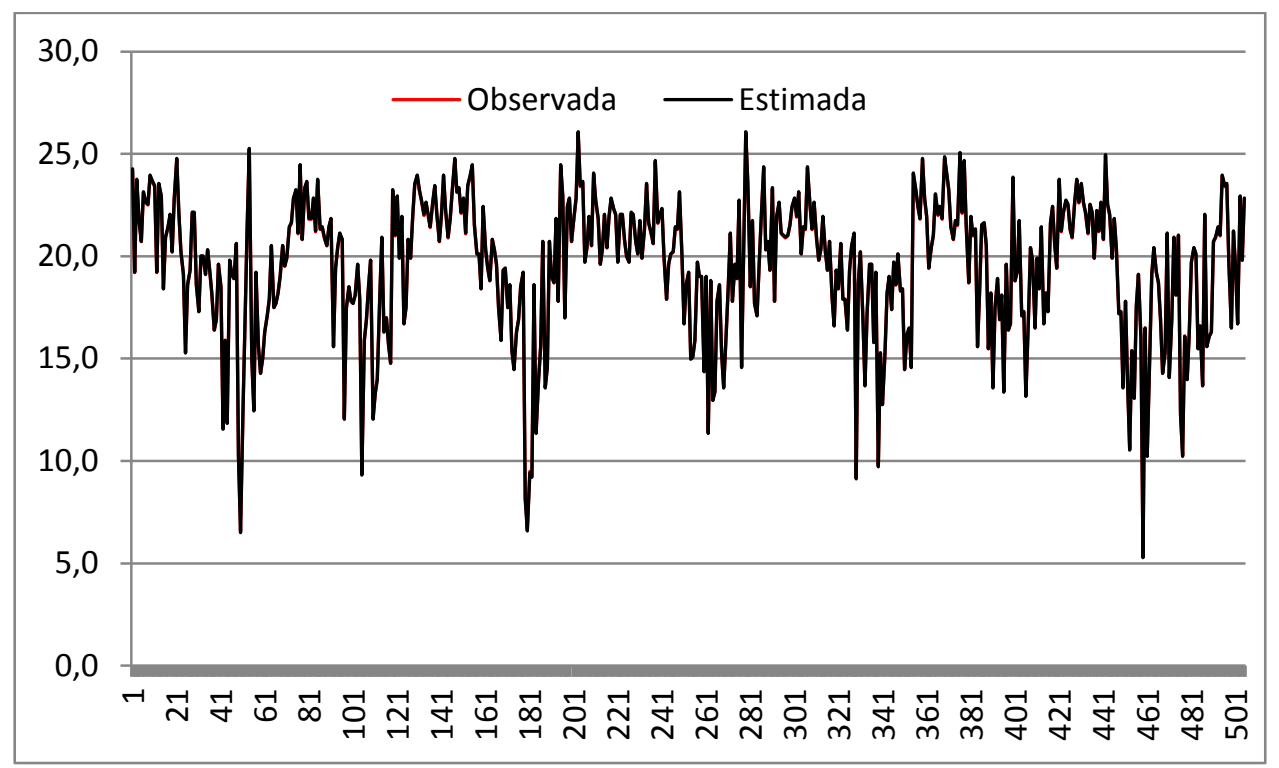

Figura 2: Gráfico dos valores observados e estimados pelo modelo neuro-fuzzy para o conjunto de dados analisado

\section{Conclusões}

A metodologia gerou modelos neuro-fuzzy simples com boa capacidade de generalização; os modelos obtidos podem ser utilizados para a estimativa da temperatura do ar com boa eficiência; os valores obtidos pelo modelo neuro-fuzzy e os valores observados estão bem correlacionados $(r=0.999)$.

\section{Referências}

[1] Chiu, S.L. Selecting input variables for fuzzy models. Journal of Intelligent and Fuzzy Systems, vol.4, n.4, pp. 243-256, (1996).

[2] Jang, J-R.R.,Adaptive-network based fuzzy inference systems. IEEE Transactions on Systems, Man and Cybernetics, vol.23, n.3: pp. 665-685, (1993).

[3] Takagi, T. ; Sugeno, M. , Fuzzy identification of systems and its applications to modelling and control. IEEE Transactions on Systems, Man and Cybernetics, vol.15, n.1, pp. 116-132, (1985).

[4] Zadeh, L.A., Fuzzy sets. Information and control, vol. 8:29-44, (1965).

[5] ZAdeh,L.A., Outline of a new approach to the analysis of complex systems and decision processes. IEEE Transactions on Systems, Man, and Cybernetics, vol.3, n.1: pp. 28-44,. (1973). 